Open J. Math. Anal., Vol. 2(2018), No. 1, pp. $33-46$

Website: https://pisrt.org/psr-press/journals/oma/

ISSN: 2616-8111 (Online) 2616-8103 (Print)

http://dx.doi.org/10.30538/psrp-oma2018.0010

\title{
NEW TYPE INTEGRAL INEQUALITIES FOR THREE TIMES DIFFERENTIABLE PREINVEX AND PREQUASIINVEX FUNCTIONS
}

\author{
HURIYE KADAKAL ${ }^{1}$, MAHIR KADAKAL, IMDAT IŞCAN
}

\begin{abstract}
In this paper, a new identity for functions defined on an open invex subset of set of real numbers is established, and by using the this identity and the Hölder and Power mean integral inequalities we present new type integral inequalities for functions whose powers of third derivatives in absolute value are preinvex and prequasiinvex functions.
\end{abstract}

AMS Mathematics Subject Classification: 26A51, 26D10, $26 \mathrm{D} 15$.

Key words and phrases: invex set, preinvex function, prequasiinvex function, Hölder integral inequality

\section{Preliminaries}

Definition 1.1. A function $f: I \subseteq \mathbb{R} \rightarrow \mathbb{R}$ is said to be convex if the inequality $f(t x+(1-t) y) \leq t f(x)+(1-t) f(y)$ is valid for all $x, y \in I$ and $t \in[0,1]$. If this inequality reverses, then $f$ is said to be concave on interval $I \neq \varnothing$.

This definition is well known in the literature. Convexity theory has appeared as a powerful technique to study a wide class of unrelated problems in pure and applied sciences.

Definition 1.2. $f: I \subseteq \mathbb{R} \rightarrow \mathbb{R}$ be a convex function on the interval $I$ of real numbers and $a, b \in I$ with $a<b$. The following celebrated double inequalityis well known in the literature as Hermite-Hadamard's inequality for convex functions [1]. Both inequalities hold in the reserved direction if $f$ is concave.

Received 10-01-2018. Revised 21-03-2018.

${ }^{1}$ Corresponding Author

(C) 2018 Huriye Kadakal, Mahir Kadakal, İmdat İşcan. This is an open access article distributed under the Creative Commons Attribution License, which permits unrestricted use, distribution, and reproduction in any medium, provided the original work is properly cited. 
The classical Hermite-Hadamard inequality provides estimates of the mean value of a continuous convex or concave function. Hadamard's inequality for convex or concave functions has received renewed attention in recent years and a remarkable variety of refinements and generalizations have been found; for example see [1, 2, 3, 4, 5]. Hermite-Hadamard inequality (see [6]) has been considered the most useful inequality in mathematical analysis. Some of the classical inequalities for means can be derived from Hermite-Hadamard inequality for particular choices of the function $f$.

Definition 1.3. A function $f: I \subseteq \mathbb{R} \rightarrow \mathbb{R}$ is said to be quasi-convex if the inequality $f(t x+(1-t) y) \leq \max \{f(x), f(y)\}$ holds for all $x, y \in I$ and $t \in$ $[0,1]$. Clearly, any convex function is a quasi-convex function. Furthermore, there exist quasi-convex functions which are not convex [7].

Let us recall the notions of preinvexity and prequasiinvexity which are signicant generalizations of the notions of convexity and qusi-convexity respectively, and some related results.

Definition 1.4 (8]). Let $K$ be a non-empty subset in $\mathbb{R}^{n}$ and $\eta: K \times K \rightarrow \mathbb{R}^{n}$. Let $x \in K$, then the set $K$ is said to be invex at $x$ with respect to $\eta(\cdot, \cdot)$, if $x+t \eta(y, x) \in K, \forall x, y \in K t \in[0,1] . K$ is said to be an invex set with respect to $\eta$ if $K$ is invex at each $x \in K$. The invex set $K$ is also called $\eta$-connected set.

Definition 1.4 essentially says that there is a path starting from a point $x$ which is contained in $K$. We do not require that the point $y$ should be one of the end points of the path. This observation plays an important role in our analysis. Note that, if we demand that $y$ should be an end point of the path for every pair of points $x, y \in K$, then $\eta(y, x)=y-x$, and consequently invexity reduces to convexity. Thus, it is true that every convex set is also an invex set with respect to $\eta(y, x)=y-x$, but the converse is not necessarily true, see [9, 10] and the references therein. For the sake of simplicity, we always assume that $K=[x, x+t \eta(y, x)]$, unless otherwise specified [11].

Definition $1.5([])$. A function $f: K \rightarrow \mathbb{R}$ on an invex set $K \subseteq \mathbb{R}$ is said to be preinvex with respect to $\eta$, if $f(u+t \eta(v, u)) \leq(1-t) f(u)+t f(v), \forall u, v \in K, t \in$ $[0,1]$. The function $f$ is said to be preconcave if and only if $-f$ is preinvex.

It is to be noted that every convex function is preinvex with respect to the map $\eta(y, x)=x-y$ but the converse is not true see for instance.

Definition 1.6 ([12]). A function $f: K \rightarrow \mathbb{R}$ on an invex set $K \subseteq \mathbb{R}$ is said to be prequasiinvex with respect to $\eta$, if $f(u+t \eta(v, u)) \leq \max \{f(u), f(v)\}, \forall u, v \in$ $K, t \in[0,1]$.

Also every quasi-convex function is a prequasiinvex with respect to the map $\eta(v, u)=v-u$ but the converse does not hold, see for example [12. Mohan and Neogy [9] introduced Condition $C$ defined as follows: 
Definition $1.7([9])$. Let $S \subseteq \mathbb{R}$ be an open invex subset with respect to the map $\eta: S \times S \rightarrow \mathbb{R}$. We say that the function satisfies the Condition $C$ if, for any $x, y \in S$ and any $t \in[0,1]$,

$$
\begin{gathered}
\eta(y, y+t \eta(x, y))=-t \eta(x, y) \\
\eta(x, y+t \eta(x, y))=(1-t) \eta(x, y) .
\end{gathered}
$$

Note that, from the Condition $C$, we have

$$
\eta\left(y+t_{2} \eta(x, y), y+t_{1} \eta(x, y)\right)=\left(t_{2}-t_{1}\right) \eta(x, y)
$$

for any $x, y \in S$ and any $t_{1}, t_{2} \in[0,1]$.

In recent years, many mathematicians have been studying about preinvexity and types of preinvexity. A lot of efforts have been made by many mathematicians to generalize the classical convexity. These studies include, among others, the work of $[8,13,14,15,16,17$. In this papers have been studied the basic properties of the preinvex functions and their role in optimization, variational inequalities and equilibrium problems. Ben-Israel and Mond gave the concept of preinvex functions which is a special case of invexity [13. Pini introduced the concept of prequasiinvex functions as a generalization of invex functions [16. In a recent paper, Noor has obtained the following Hermite-Hadamard type inequalities for the preinvex functions [18.

Theorem 1.8 ([18]). Let $f:[a, a+t \eta(b, a)] \rightarrow(0, \infty)$ be a preinvex function on the interval of the real numbers $K^{\circ}$ (the interior of $K$ ) and $a, b \in K^{\circ}$ with $\eta(b, a)>0$. Then the following inequalities holds

$$
f\left(\frac{2 a+\eta(b, a)}{2}\right) \leq \frac{1}{\eta(b, a)} \int_{a}^{a+\eta(b, a)} f(x) d x \leq \frac{f(a)+f(b)}{2}
$$

For several recent results on inequalities for preinvex and prequasiinvex functions which are connected to (3), we refer the interested reader to [19, 20, 21, 22] and the references therein. Let $0<a<b$, throughout this paper we will use

$$
\begin{aligned}
A & =A(a, b)=\frac{a+b}{2} \\
L_{p}(a, b) & =\left(\frac{b^{p+1}-a^{p+1}}{(p+1)(b-a)}\right)^{\frac{1}{p}}, a \neq b, p \in \mathbb{R}, \quad p \neq-1,0
\end{aligned}
$$

for the arithmetic and generalized logarithmic mean, respectively. Moreover, for shortness, the following notations will be used:

$$
\begin{aligned}
& \alpha=\alpha(a, b, \eta)=a+\frac{\eta(b, a)}{2}, \quad \alpha_{t}=\alpha_{t}(a, b, \eta)=a+t \frac{\eta(b, a)}{2}, \\
& \beta=\beta(a, b, \eta)=a+\frac{\eta(b, a)}{3}, \quad \beta_{t}=\beta_{t}(a, b, \eta)=a+t \frac{\eta(b, a)}{3},
\end{aligned}
$$

and

$$
I_{f}(a, b, \eta):=\frac{\eta^{2}(b, a)}{2}\left(a+\frac{\eta(b, a)}{3}\right) f^{\prime \prime}(a+\eta(b, a))
$$




$$
\begin{aligned}
& -\eta(b, a)\left(a+\frac{\eta(b, a)}{2}\right) f^{\prime}(a+\eta(b, a)) \\
& +f(a+\eta(b, a))(a+\eta(b, a))-f(a) a-\int_{a}^{a+\eta(b, a)} f(x) d x .
\end{aligned}
$$

In this paper, using a general integral identity for a three times differentiable functions, we establish some new type integral inequalities for mappings whose third derivative in absolute value at certain powers are preinvex and prequasiinvex.

\section{Main Results}

We will use the following Lemmas for obtain our main results about the preinvexity and prequasiinvexity.

Lemma 2.1. Let $K \subseteq \mathbb{R}$ be an open invex subset with respect to mapping $\eta(\cdot, \cdot)$ : $K \times K \rightarrow \mathbb{R}^{n}$ and $a, b \in K$ with $\eta(b, a)>0$. Suppose that the function $f: K \rightarrow \mathbb{R}$ is a three times differentiable function on $K$ such that $f^{\prime \prime \prime} \in L[a, a+\eta(b, a)]$. Then the following identity hold:

$$
\begin{aligned}
& \frac{\eta^{2}(b, a)}{2} \beta f^{\prime \prime}(a+\eta(b, a))-\eta(b, a) \alpha f^{\prime}(a+\eta(b, a)) \\
& +f(a+\eta(b, a))(a+\eta(b, a))-f(a) a-\int_{a}^{a+\eta(b, a)} f(x) d x \\
= & \eta^{3}(b, a) \int_{0}^{1} \frac{t^{2}}{2} \beta_{t} f^{\prime \prime \prime}(a+t \eta(b, a)) d t .
\end{aligned}
$$

Proof. Integrating three times by parts and then changing the variable, we obtain

$$
\begin{aligned}
& \eta^{3}(b, a) \int_{0}^{1} \frac{t^{2}}{2} \beta_{t} f^{\prime \prime \prime}(a+t \eta(b, a)) d t \\
= & \left.\eta^{2}(b, a) \frac{t^{2}}{2} \beta_{t} f^{\prime \prime}(a+t \eta(b, a))\right|_{0} ^{1}-\left.\eta(b, a) t \alpha_{t} f^{\prime}(a+t \eta(b, a))\right|_{0} ^{1} \\
& +\left.(a+t \eta(b, a)) f(a+t \eta(b, a))\right|_{0} ^{1}-\eta(b, a) \int_{0}^{1} f(a+t \eta(b, a)) d t \\
= & \frac{\eta^{2}(b, a)}{2} \beta f^{\prime \prime}(a+\eta(b, a))-\eta(b, a) \alpha f^{\prime}(a+\eta(b, a)) \\
& +(a+\eta(b, a)) f(a+\eta(b, a))-a f(a)-\eta(b, a) \int_{0}^{1} f(a+t \eta(b, a)) d t \\
= & \frac{\eta^{2}(b, a)}{2} \beta f^{\prime \prime}(a+\eta(b, a))-\eta(b, a) \alpha f^{\prime}(a+\eta(b, a)) \\
& +(a+\eta(b, a)) f(a+\eta(b, a))-a f(a)-\int_{a}^{a+\eta(b, a)} f(x) d x .
\end{aligned}
$$

This completes the proof of lemma. 
Theorem 2.2. Let $K \subseteq \mathbb{R}$ be an open invex subset with respect to mapping $\eta(\cdot, \cdot): K \times K \rightarrow \mathbb{R}^{n}$ and $a, b \in K$ with $\eta(b, a)>0$. Suppose that the function $f: K \rightarrow \mathbb{R}$ is a three times differentiable function on $K$ such that $f^{\prime \prime \prime} \in L[a, a+\eta(b, a)]$. If $\left|f^{\prime \prime \prime}\right|^{q}$ is preinvex on $K$ for $q>1$, then the following inequality holds:

$$
\left|I_{f}(a, b, \eta)\right| \leq \frac{3^{\frac{1}{q}}}{2} \frac{\eta^{1+\frac{2}{p}}(b, a)}{(2 p+1)^{\frac{1}{p}}}\left[\left|f^{\prime \prime \prime}(b)\right|^{q} C_{1, \eta}(a, b)+\left|f^{\prime \prime \prime}(a)\right|^{q} C_{2, \eta}(a, b)\right]^{\frac{1}{q}} .
$$

where

$$
C_{1, \eta}(a, b):=\left\{\begin{array}{lr}
\eta(b, a)\left[L_{q+1}^{q+1}(\beta, a)-a L_{q}^{q}(\beta, a)\right], & a>0, \beta>0, \\
3(a+\beta) L_{q+1}^{q+1}(\beta,-a)-\frac{6 a}{q+1} A\left(\beta^{q+1},(-a)^{q+1}\right), & a<0, \beta>0, \\
-\eta(b, a)\left[L_{q+1}^{q+1}(-a,-\beta)+a L_{q}^{q}(-a,-\beta)\right], & a<0, \beta<0 .
\end{array}\right.
$$

and

$$
C_{2, \eta}(a, b):=\left\{\begin{array}{lr}
-\eta(b, a)\left[L_{q+1}^{q+1}(\beta, a)-\alpha L_{q}^{q}(\beta, a)\right], & a>0, \beta>0, \\
-3(a+\beta) L_{q+1}^{q+1}(\beta,-a)+\frac{6 \beta}{q+1} A\left(\beta^{q+1},(-a)^{q+1}\right), & a<0, \beta>0, \\
\eta(b, a)\left[L_{q+1}^{q+1}(-a,-\beta)+\alpha L_{q}^{q}(-a,-\beta)\right], & a<0, \beta<0 .
\end{array}\right.
$$

Proof. If $\left|f^{\prime \prime \prime}\right|^{q}$ for $q>1$ is preinvex on $[a, a+\eta(b, a)]$, using Lemma 2.1, the Hölder integral inequality and $\left|f^{\prime \prime \prime}(a+t \eta(b, a))\right|^{q} \leq t\left|f^{\prime \prime \prime}(b)\right|^{q}+(1-t)\left|f^{\prime \prime \prime}(a)\right|^{q}$, we get

$$
\begin{aligned}
& \left|I_{f}(a, b, \eta)\right| \\
\leq & \frac{1}{2} \eta^{3}(b, a) \int_{0}^{1} t^{2}\left|\beta_{t}\right|\left|f^{\prime \prime \prime}(a+t \eta(b, a))\right| d t \\
\leq & \frac{1}{2} \eta^{3}(b, a)\left(\int_{0}^{1} t^{2 p} d t\right)^{\frac{1}{p}}\left(\int_{0}^{1}\left|\beta_{t}\right|^{q}\left|f^{\prime \prime \prime}(a+t \eta(b, a))\right|^{q} d t\right)^{\frac{1}{q}} \\
\leq & \frac{1}{2} \eta^{3}(b, a)\left(\int_{0}^{1} t^{2 p} d t\right)^{\frac{1}{p}}\left(\int_{0}^{1}\left|\beta_{t}\right|^{q}\left[t\left|f^{\prime \prime \prime}(b)\right|^{q}+(1-t)\left|f^{\prime \prime \prime}(a)\right|^{q}\right] d t\right)^{\frac{1}{q}} \\
\leq & \frac{1}{2} \frac{\eta^{3}(b, a)}{(2 p+1)^{\frac{1}{p}}}\left(\left|f^{\prime \prime \prime}(b)\right|^{q} \int_{0}^{1} t\left|\beta_{t}\right|^{q} d t+\left|f^{\prime \prime \prime}(a)\right|^{q} \int_{0}^{1}(1-t)\left|\beta_{t}\right|^{q} d t\right)^{\frac{1}{q}} \\
= & \frac{1}{2} \frac{\eta^{3}(b, a)}{(2 p+1)^{\frac{1}{p}}}\left(\frac{3\left|f^{\prime \prime \prime}(b)\right|^{q}}{\eta^{2}(b, a)} \int_{a}^{\beta} 3(x-a)|x|^{q} d x\right. \\
& \left.+\frac{3\left|f^{\prime \prime \prime}(a)\right|^{q}}{\eta^{2}(b, a)} \int_{a}^{\beta}(\eta(b, a)-3(x-a))|x|^{q} d x\right)^{\frac{1}{q}} \\
= & \frac{1}{2} 3^{\frac{1}{q}} \frac{\eta^{1+\frac{2}{p}}(b, a)}{(2 p+1)^{\frac{1}{p}}}\left(\left|f^{\prime \prime \prime}(b)\right|^{q} \int_{a}^{\beta} 3(x-a)|x|^{q} d x\right.
\end{aligned}
$$




$$
\begin{aligned}
& \left.+\left|f^{\prime \prime \prime}(a)\right|^{q} \int_{a}^{\beta}(\eta(b, a)-3(x-a))|x|^{q} d x\right)^{\frac{1}{q}} \\
= & \frac{3^{\frac{1}{q}}}{2} \frac{\eta^{1+\frac{2}{p}}(b, a)}{(2 p+1)^{\frac{1}{p}}}\left[\left|f^{\prime \prime \prime}(b)\right|^{q} C_{1, \eta}(a, b)+\left|f^{\prime \prime \prime}(a)\right|^{q} C_{2, \eta}(a, b)\right]^{\frac{1}{q}} .
\end{aligned}
$$

This completes the proof of theorem.

Corollary 2.3. Suppose that all the assumptions of Theorem 2.2 are satisfied. If we choose $\eta(b, a)=b-a$ then when $\left|f^{\prime \prime \prime}\right|^{q}$ is convex on $K$ for $q>1$ we obtain following inequality:

$$
\begin{aligned}
& \left|\frac{b-a}{2}\left(\frac{2 a+b}{3}\right) f^{\prime \prime}(b)-\frac{a+b}{2} f^{\prime}(b)+\frac{f(b) b-f(a) a}{b-a}-\frac{1}{b-a} \int_{a}^{b} f(x) d x\right| \\
\leq & \frac{1}{2} 3^{\frac{1}{q}} \frac{(b-a)^{\frac{2}{p}}}{(2 p+1)^{\frac{1}{p}}}\left[\left|f^{\prime \prime \prime}(b)\right|^{q} C_{1}(a, b)+\left|f^{\prime \prime \prime}(a)\right|^{q} C_{2}(a, b)\right]^{\frac{1}{q}},
\end{aligned}
$$

where

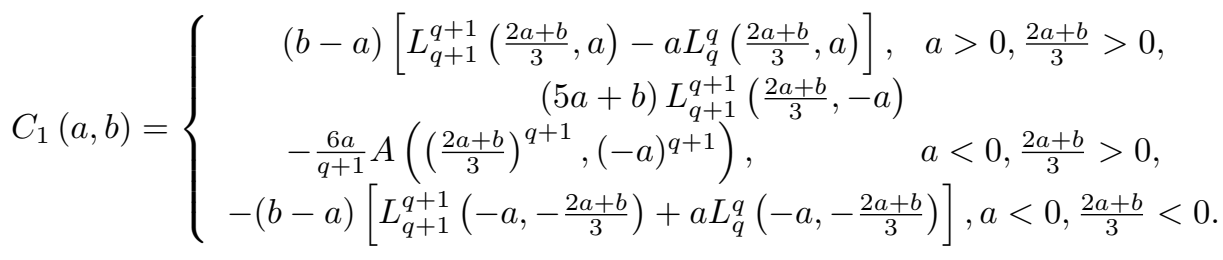

$$
\begin{aligned}
& C_{2}(a, b)=\left\{\begin{array}{c}
-(b-a)\left[L_{q+1}^{q+1}\left(\frac{2 a+b}{3}, a\right)-\alpha L_{q}^{q}\left(\frac{2 a+b}{3}, a\right)\right], \quad a>0, \frac{2 a+b}{3}>0, \\
-(5 a+b) L_{q+1}^{q+1}\left(\frac{2 a+b}{3},-a\right) \\
+\frac{6 \beta}{q+1} A\left(\left(\frac{2 a+b}{3}\right)^{q+1},(-a)^{q+1}\right)^{2}, a<0, \frac{2 a+b}{3}>0, \\
(b-a)\left[L_{q+1}^{q+1}\left(-a,-\frac{2 a+b}{3}\right)+\alpha L_{q}^{q}\left(-a,-\frac{2 a+b}{3}\right)\right], \quad a<0, \frac{2 a+b}{3}<0 .
\end{array}\right.
\end{aligned}
$$

Remark 2.4. If the mapping $\eta$ satisfies condition $\mathrm{C}$ then by use of the preinvexity of $\left|f^{\prime \prime \prime}\right|^{q}$ we obtain following inequality for every $t \in[0,1]$ :

$$
\begin{aligned}
\left|f^{\prime \prime \prime}(a+t \eta(b, a))\right|^{q} & =\left|f^{\prime \prime \prime}(a+\eta(b, a)+(1-t) \eta(a, a+\eta(b, a)))\right|^{q} \\
& \leq t\left|f^{\prime \prime \prime}(a+\eta(b, a))\right|^{q}+(1-t)\left|f^{\prime \prime \prime}(a)\right|^{q} .
\end{aligned}
$$

If we use the inequality (5) in the proof of Theorem 2.2, then the inequality (4) becomes the following inequality:

$$
\begin{aligned}
& I_{f}(a, b, \eta) \\
\leq & \frac{3^{\frac{1}{q}}}{2} \frac{\eta^{1+\frac{2}{p}}(b, a)}{(2 p+1)^{\frac{1}{p}}}\left[\left|f^{\prime \prime \prime}(a+\eta(b, a))\right|^{q} C_{1, \eta}(a, b)+\left|f^{\prime \prime \prime}(a)\right|^{q} C_{2, \eta}(a, b)\right]^{\frac{1}{q}} .
\end{aligned}
$$

We note that by use of the preinvexity of $\left|f^{\prime \prime \prime}\right|^{q}$ we get $\left|f^{\prime \prime \prime}(a+\eta(b, a))\right|^{q} \leq$ $\left|f^{\prime \prime \prime}(b)\right|^{q}$. Therefore, the inequality (6) is better than the inequality (4). 
Theorem 2.5. Let $K \subseteq \mathbb{R}$ be an open invex subset with respect to mapping $\eta(\cdot, \cdot): K \times K \rightarrow \mathbb{R}^{n}$ and $a, b \in K$ with $\eta(b, a)>0$. Suppose that the function $f: K \rightarrow \mathbb{R}$ is a three times differentiable function on $K$ such that $f^{\prime \prime \prime} \in L[a, a+\eta(b, a)]$. If $\left|f^{\prime \prime \prime}\right|^{q}$ is preinvex on $K$ for $q>1$, then the following inequality holds:

$$
\left|I_{f}(a, b, \eta)\right| \leq \frac{3^{\frac{1}{p}}}{2} \eta^{2+\frac{1}{q}}(b, a) C_{3, \eta}^{\frac{1}{p}}(a, b)\left[\frac{(2 q+1)\left|f^{\prime \prime \prime}(b)\right|^{q}+\left|f^{\prime \prime \prime}(a)\right|^{q}}{(2 q+1)(2 q+2)}\right]^{\frac{1}{q}}
$$

where

$$
C_{3, \eta}(a, b)=\left\{\begin{array}{lr}
\frac{\eta(b, a)}{3} L_{p}^{p}(\beta, a), & a>0, \beta>0, \\
\frac{2}{p+1} A\left(\beta^{p+1},(-a)^{p+1}\right), & a<0, \beta>0, \\
\frac{\eta(b, a)}{3} L_{p}^{p}(-a,-\beta), & a<0, \beta<0 .
\end{array}\right.
$$

Proof. If $\left|f^{\prime \prime \prime}\right|^{q}$ for $q>1$ is preinvex on $[a, a+\eta(b, a)]$, using Lemma 2.1, the Hölder integral inequality and $\left|f^{\prime \prime \prime}(a+t \eta(b, a))\right|^{q} \leq t\left|f^{\prime \prime \prime}(b)\right|^{q}+(1-t)\left|f^{\prime \prime \prime}(a)\right|^{q}$, we obtain the following inequality:

$$
\begin{aligned}
&\left|I_{f}(a, b, \eta)\right| \\
& \leq \frac{1}{2} \eta^{3}(b, a) \int_{0}^{1} t^{2}\left|\beta_{t}\right|\left|f^{\prime \prime \prime}(a+t \eta(b, a))\right| d t \\
& \leq \frac{1}{2} \eta^{3}(b, a)\left(\int_{0}^{1}\left|\beta_{t}\right|^{p} d t\right)^{\frac{1}{p}}\left(\int_{0}^{1} t^{2 q}\left|f^{\prime \prime \prime}(a+t \eta(b, a))\right|^{q} d t\right)^{\frac{1}{q}} \\
& \leq \frac{1}{2} \eta^{3}(b, a)\left(\int_{0}^{1}\left|\beta_{t}\right|^{p} d t\right)^{\frac{1}{p}} \\
& \times\left(\int_{0}^{1} t^{2 q}\left[t\left|f^{\prime \prime \prime}(b)\right|^{q}+(1-t)\left|f^{\prime \prime \prime}(a)\right|^{q}\right] d t\right)^{\frac{1}{q}} \\
&= \frac{1}{2} \eta^{3}(b, a)\left(\int_{0}^{1}\left|\beta_{t}\right|^{p} d t\right)^{\frac{1}{p}} \\
& \times\left(\left|f^{\prime \prime \prime}(b)\right|^{q} \int_{0}^{1} t^{2 q+1} d t+\left|f^{\prime \prime \prime}(a)\right|^{q} \int_{0}^{1}\left(t^{2 q}-t^{2 q+1}\right) d t\right)^{\frac{1}{q}} \\
&=\frac{1}{2} 3^{\frac{1}{p}} \eta^{2+\frac{1}{q}}(b, a)\left(\int_{a}^{\beta}|x|^{p} d x\right)^{\frac{1}{p}}\left[\frac{\left|f^{\prime \prime \prime}(b)\right|^{q}}{2 q+2}+\left|f^{\prime \prime \prime}(a)\right|^{q}\left(\frac{1}{2 q+1}-\frac{1}{2 q+2}\right)\right]^{\frac{1}{q}} \\
& \frac{1}{2} 3^{\frac{1}{p}} \eta^{2+\frac{1}{q}}(b, a) C_{3, \eta}^{\frac{1}{p}}(a, b)\left[\frac{(2 q+1)\left|f^{\prime \prime \prime}(b)\right|^{q}+\left|f^{\prime \prime \prime}(a)\right|^{q}}{(2 q+1)(2 q+2)}\right]^{\frac{1}{q}} \cdot
\end{aligned}
$$

This completes the proof of theorem.

Corollary 2.6. Suppose that all the assumptions of Theorem 2.5 are satisfied. If we choose $\eta(b, a)=b-a$ then when $\left|f^{\prime \prime \prime}\right|^{q}$ is convex on $K$ for $q>1$ we have 
the following inequality:

$$
\begin{aligned}
& \left|\frac{b-a}{2}\left(\frac{2 a+b}{3}\right) f^{\prime \prime}(b)-\frac{a+b}{2} f^{\prime}(b)+\frac{f(b) b-f(a) a}{b-a}-\frac{1}{b-a} \int_{a}^{b} f(x) d x\right| \\
\leq & \frac{3^{\frac{1}{p}}}{2}(b-a)^{1+\frac{1}{q}} C_{3}^{\frac{1}{p}}(a, b)\left[\frac{(2 q+1)\left|f^{\prime \prime \prime}(b)\right|^{q}+\left|f^{\prime \prime \prime}(a)\right|^{q}}{(2 q+1)(2 q+2)}\right]^{\frac{1}{q}},
\end{aligned}
$$

where

$$
C_{3}(a, b)=\left\{\begin{array}{lr}
\frac{b-a}{3} L_{p}^{p}\left(\frac{2 a+b}{3}, a\right), & a>0, \frac{2 a+b}{3}>0, \\
\frac{2}{p+1} A\left(\left(\frac{2 a+b}{3}\right)^{p+1},(-a)^{p+1}\right), & a<0, \frac{2 a+b}{3}>0, \\
\frac{b-a}{3} L_{p}^{p}\left(-a,-\frac{2 a+b}{3}\right), & a<0, \frac{2 a+b}{3}<0 .
\end{array}\right.
$$

Remark 2.7. If the mapping $\eta$ satisfies condition $\mathrm{C}$ then using the inequality (5) in the proof of Theorem 2.5. then the inequality (7) becomes the following inequality:

$$
\left|I_{f}(a, b, \eta)\right| \leq \frac{3^{\frac{1}{p}}}{2} \eta^{2+\frac{1}{q}}(b, a) C_{3, \eta}^{\frac{1}{p}}(a, b)\left[\frac{(2 q+1)\left|f^{\prime \prime \prime}(a+\eta(b, a))\right|^{q}+\left|f^{\prime \prime \prime}(a)\right|^{q}}{(2 q+1)(2 q+2)}\right]^{\frac{1}{q}} .
$$

We note that by use of the preinvexity of $\left|f^{\prime \prime \prime}\right|^{q}$ we get $\left|f^{\prime \prime \prime}(a+\eta(b, a))\right|^{q} \leq$ $\left|f^{\prime \prime \prime}(b)\right|^{q}$. Therefore, the inequality (8) is better than the inequality (7).

Theorem 2.8. Let $K \subseteq \mathbb{R}$ be an open invex subset with respect to mapping $\eta(\cdot, \cdot): K \times K \rightarrow \mathbb{R}^{n}$ and $a, b \in K$ with $\eta(b, a)>0$. Suppose that the function $f: K \rightarrow \mathbb{R}$ is a three times differentiable function on $K$ such that $f^{\prime \prime \prime} \in L[a, a+\eta(b, a)]$. If $\left|f^{\prime \prime \prime}\right|^{q}$ is preinvex on $K$ for $q \geq 1$, then the following inequality holds:

$$
\left|I_{f}(a, b, \eta)\right| \leq \frac{27}{2} \eta^{-\frac{1}{q}}(b, a) D_{1, \eta}^{1-\frac{1}{q}}(a, b)\left[\left|f^{\prime \prime \prime}(b)\right|^{q} D_{2, \eta}(a, b)+\left|f^{\prime \prime \prime}(a)\right|^{q} D_{3, \eta}(a, b)\right]^{\frac{1}{q}}
$$

where

$$
\begin{aligned}
& D_{1, \eta}(a, b):= \begin{cases}\frac{\eta^{3}(b, a)}{27} \frac{\eta(b, a)+4 a}{12}, & a>0, \beta>0 \\
\frac{\eta^{3}(b, a)}{27} \frac{\eta(b, a)+4 a}{12}+\frac{a^{4}}{6}, a<0, \beta>0 & , \quad \\
-\frac{\eta^{3}(b, a)}{27} \frac{\eta(b, a)+4 a}{12}, & a<0, \beta<0\end{cases} \\
& D_{2, \eta}(a, b):= \begin{cases}\frac{\eta^{4}(b, a)}{27} \frac{4 \eta(b, a)+15 a}{60}, & a>0, \beta>0 \\
\frac{\eta^{4}(b, a)}{27} \frac{4 \eta(b, a)+15 a}{60}-3 \frac{a^{5}}{10}, a<0, \beta>0, & a<0, \beta<0 \\
-\frac{\eta^{4}(b, a)}{27} \frac{4 \eta(b, a)+15 a}{60}, & a>0, \beta>0\end{cases} \\
& D_{3, \eta}(a, b):= \begin{cases}\frac{\eta^{4}(b, a)}{27} \frac{\eta(b, a)+5 a}{60}, & a<0, \beta<0 . \\
\frac{\eta^{4}(b, a)}{27} \frac{\eta(b, a)+5 a}{60}+\eta(b, a) \frac{a^{4}}{6}+3 \frac{a^{5}}{10}, & a<0, \beta>0 \\
-\frac{\eta^{4}(b, a)}{27} \frac{\eta(b, a)+5 a}{60}, & a<0\end{cases}
\end{aligned}
$$


Proof. Using Lemma 2.1 and Power-mean integral inequality, we obtain

$$
\begin{aligned}
& \left|I_{f}(a, b, \eta)\right| \\
& \leq \frac{1}{2} \eta^{3}(b, a) \int_{0}^{1} t^{2}\left|\beta_{t}\right|\left|f^{\prime \prime \prime}(a+t \eta(b, a))\right| d t \\
& \leq \frac{1}{2} \eta^{3}(b, a)\left(\int_{0}^{1} t^{2}\left|\beta_{t}\right| d t\right)^{1-\frac{1}{q}}\left(\int_{0}^{1} t^{2}\left|\beta_{t}\right|\left|f^{\prime \prime \prime}(a+t \eta(b, a))\right|^{q} d t\right)^{\frac{1}{q}} \\
& \leq \frac{1}{2} \eta^{3}(b, a)\left(\int_{0}^{1} t^{2}\left|\beta_{t}\right| d t\right)^{1-\frac{1}{q}} \\
& \times\left(\int_{0}^{1} t^{2}\left|\beta_{t}\right|\left[t\left|f^{\prime \prime \prime}(b)\right|^{q}+(1-t)\left|f^{\prime \prime \prime}(a)\right|^{q}\right] d t\right)^{\frac{1}{q}} \\
& =\frac{1}{2} \eta^{3}(b, a)\left(\int_{0}^{1} t^{2}\left|\beta_{t}\right| d t\right)^{1-\frac{1}{q}} \\
& \times\left(\left|f^{\prime \prime \prime}(b)\right|^{q} \int_{0}^{1} t^{3}\left|\beta_{t}\right| d t+\left|f^{\prime \prime \prime}(a)\right|^{q} \int_{0}^{1} t^{2}(1-t)\left|\beta_{t}\right| d t\right)^{\frac{1}{q}} \\
& =\frac{1}{2} \eta^{3}(b, a)\left(\frac{27}{\eta^{3}(b, a)}\right)^{1-\frac{1}{q}}\left(\frac{27}{\eta^{4}(b, a)}\right)^{\frac{1}{q}}\left(\int_{a}^{\beta}(x-a)^{2}|x| d x\right)^{1-\frac{1}{q}} \\
& \times\left(\begin{array}{c}
\left|f^{\prime \prime \prime}(b)\right|^{q} \int_{a}^{\beta} 3(x-a)^{3}|x| d x \\
+\left|f^{\prime \prime \prime}(a)\right|^{q} \int_{a}^{\beta}(x-a)^{2}[\eta(b, a)-3(x-a)]|x| d x
\end{array}\right)^{\frac{1}{q}} \\
& =\frac{27}{2} \eta^{-\frac{1}{q}}(b, a)\left(\int_{a}^{\beta}(x-a)^{2}|x| d x\right)^{1-\frac{1}{q}} \\
& \times\left(\begin{array}{c}
\left|f^{\prime \prime \prime}(b)\right|^{q} \int_{a}^{\beta} 3(x-a)^{3}|x| d x \\
+\left|f^{\prime \prime \prime}(a)\right|^{q} \int_{a}^{\beta}(x-a)^{2}[\eta(b, a)-3(x-a)]|x| d x
\end{array}\right)^{\frac{1}{q}} \\
& =\frac{27}{2} \eta^{-\frac{1}{q}}(b, a) D_{1, \eta}^{1-\frac{1}{q}}(a, b)\left[\left|f^{\prime \prime \prime}(b)\right|^{q} D_{2, \eta}(a, b)+\left|f^{\prime \prime \prime}(a)\right|^{q} D_{3, \eta}(a, b)\right]^{\frac{1}{q}} \text {. }
\end{aligned}
$$

This completes the proof of theorem.

Corollary 2.9. Suppose that all the assumptions of Theorem 2.5 are satisfied. If we choose $\eta(b, a)=b-a$ then when $\left|f^{\prime \prime \prime}\right|^{q}$ is convex on $K$ for $q \geq 1$ we get

$$
\begin{aligned}
& \left|\frac{b-a}{2}\left(\frac{2 a+b}{3}\right) f^{\prime \prime}(b)-\frac{a+b}{2} f^{\prime}(b)+\frac{f(b) b-f(a) a}{b-a}-\frac{1}{b-a} \int_{a}^{b} f(x) d x\right| \\
\leq & \frac{27}{2}(b-a)^{-1-\frac{1}{q}} D_{1}^{1-\frac{1}{q}}(a, b)\left[\left|f^{\prime \prime \prime}(b)\right|^{q} D_{2}(a, b)+\left|f^{\prime \prime \prime}(a)\right|^{q} D_{3}(a, b)\right]^{\frac{1}{q}},
\end{aligned}
$$


where

$$
\begin{aligned}
& D_{1}(a, b)=\left\{\begin{array}{lr}
\frac{(b-a)^{3}}{27} \frac{b+3 a}{12}, & a>0, \beta>0 \\
\frac{(b-a)^{3}}{27} \frac{b+3 a}{12}+\frac{a^{4}}{6}, & a<0, \beta>0 \\
-\frac{(b-a)^{2}}{27} \frac{b+3 a}{12}, & a<0, \beta<0
\end{array},\right. \\
& D_{2}(a, b)=\left\{\begin{array}{lr}
\frac{(b-a)^{4}}{27} \frac{4 b+11 a}{60}, & a>0, \beta>0 \\
\frac{(b-a)^{4}}{27} \frac{4 b+11 a}{60}-3 \frac{a^{5}}{10}, & a<0, \beta>0 \\
-\frac{(b-a)^{4}}{27} \frac{4 b+11 a}{60}, & a<0, \beta<0
\end{array},\right.
\end{aligned}
$$

and

$$
D_{3}(a, b)=\left\{\begin{array}{lr}
\frac{(b-a)^{4}}{27} \frac{b+4 a}{60}, & a>0, \beta>0 \\
\frac{(b-a)^{4}}{27} \frac{b+4 a}{60}+(b-a) \frac{a^{4}}{6}+3 \frac{a^{5}}{10}, & a<0, \beta>0 \\
-\frac{(b-a)^{4}}{27} \frac{b+4 a}{60}, & a<0, \beta<0 .
\end{array}\right.
$$

Remark 2.10. If the mapping $\eta$ satisfies condition $\mathrm{C}$ then using the inequality (5) in the proof of Theorem 2.8, then the inequality (9) becomes the following inequality:

$$
\begin{aligned}
\left|I_{f}(a, b, \eta)\right| \leq & \frac{27}{2} \eta^{-\frac{1}{q}}(b, a) D_{1, \eta}^{1-\frac{1}{q}}(a, b) \\
& \times\left[\left|f^{\prime \prime \prime}(a+\eta(b, a))\right|^{q} D_{2, \eta}(a, b)+\left|f^{\prime \prime \prime}(a)\right|^{q} D_{3, \eta}(a, b)\right]^{\frac{1}{q}} .
\end{aligned}
$$

We note that by use of the preinvexity of $\left|f^{\prime \prime \prime}\right|^{q}$ we get $\left|f^{\prime \prime \prime}(a+\eta(b, a))\right|^{q} \leq$ $\left|f^{\prime \prime \prime}(b)\right|^{q}$. Therefore, the inequality (10) is better than the inequality $(9)$.

Corollary 2.11. If we take $q=1$ in Theorem 2.8, then we have the following inequality:

$$
\left|I_{f}(a, b, \eta)\right| \leq \frac{27}{2 \eta(b, a)}\left[\left|f^{\prime \prime \prime}(b)\right| D_{2, \eta}(a, b)+\left|f^{\prime \prime \prime}(a)\right| D_{3, \eta}(a, b)\right]
$$

Now we will give our results for prequasiinvex functions by using Lemma 2.1

Theorem 2.12. Let $K \subseteq \mathbb{R}$ be an open invex subset with respect to mapping $\eta(\cdot, \cdot): K \times K \rightarrow \mathbb{R}^{n}$ and $a, b \in K$ with $\eta(b, a)>0$. Suppose that the function $f: K \rightarrow \mathbb{R}$ is a three times differentiable function on $K$ such that $f^{\prime \prime \prime} \in L[a, a+\eta(b, a)]$. If $\left|f^{\prime \prime \prime}\right|^{q}$ is prequasiinvex on $K$ for $q>1$, then the following inequality holds:

$$
\begin{aligned}
& \left|I_{f}(a, b, \eta)\right| \\
\leq & \frac{3^{\frac{1}{q}}}{2} \eta^{2+\frac{1}{p}}(b, a)\left(\frac{1}{2 p+1}\right)^{\frac{1}{p}}\left(\max \left\{\left|f^{\prime \prime \prime}(a)\right|^{q},\left|f^{\prime \prime \prime}(b)\right|^{q}\right\}\right)^{\frac{1}{q}} C_{\eta}^{\frac{1}{q}}(q, a, b)(11)
\end{aligned}
$$

where

$$
C_{\eta}(q, a, b):=\left\{\begin{array}{lr}
\frac{\eta(b, a)}{3} L_{q}^{q}(\beta, a), & a>0, \beta>0, \\
\frac{2}{q+1} A\left[\beta^{q+1},(-a)^{q+1}\right], & a<0, \beta>0, \\
\frac{\eta(b, a)}{3} L_{q}^{q}(-a,-\beta), & a<0, \beta<0 .
\end{array}\right.
$$


Proof. If $\left|f^{\prime \prime \prime}\right|^{q}$ for $q>1$ is prequasiinvex on $[a, a+\eta(b, a)]$, using Lemma 2.1, the Hölder integral inequality and $\left|f^{\prime \prime \prime}(a+t \eta(b, a))\right|^{q} \leq \max \left\{\left|f^{\prime \prime \prime}(a)\right|^{q},\left|f^{\prime \prime \prime}(b)\right|^{q}\right\}$ we obtain

$$
\begin{aligned}
& \left|I_{f}(a, b, \eta)\right| \\
\leq & \frac{1}{2} \eta^{3}(b, a) \int_{0}^{1} t^{2}\left|\beta_{t}\right|\left|f^{\prime \prime \prime}(a+t \eta(b, a))\right| d t \\
\leq & \frac{1}{2} \eta^{3}(b, a)\left(\int_{0}^{1} t^{2 p} d t\right)^{\frac{1}{p}}\left(\int_{0}^{1}\left|\beta_{t}\right|^{q}\left|f^{\prime \prime \prime}(a+t \eta(b, a))\right|^{q} d t\right)^{\frac{1}{q}} \\
\leq & \frac{1}{2} \eta^{3}(b, a)\left(\int_{0}^{1} t^{2 p} d t\right)^{\frac{1}{p}}\left(\int_{0}^{1}\left|\beta_{t}\right|^{q} \max \left\{\left|f^{\prime \prime \prime}(a)\right|^{q},\left|f^{\prime \prime \prime}(b)\right|^{q}\right\} d t\right)^{\frac{1}{q}} \\
= & \frac{3^{\frac{1}{q}}}{2} \eta^{2+\frac{1}{p}}(b, a)\left(\frac{1}{2 p+1}\right)^{\frac{1}{p}}\left(\max \left\{\left|f^{\prime \prime \prime}(a)\right|^{q},\left|f^{\prime \prime \prime}(b)\right|^{q}\right\}\right)^{\frac{1}{q}}\left(\int_{a}^{\beta}|x|^{q} d x\right)^{\frac{1}{q}} \\
= & \frac{3^{\frac{1}{q}}}{2} \eta^{2+\frac{1}{p}}(b, a)\left(\frac{1}{2 p+1}\right)^{\frac{1}{p}}\left(\max \left\{\left|f^{\prime \prime \prime}(a)\right|^{q},\left|f^{\prime \prime \prime}(b)\right|^{q}\right\}\right)^{\frac{1}{q}} C_{\eta}^{\frac{1}{q}}(q, a, b)
\end{aligned}
$$

This completes the proof of theorem.

Corollary 2.13. Suppose that all the assumptions of Theorem 2.12 are satisfied. If we choose $\eta(b, a)=b-a$ then when $\left|f^{\prime \prime \prime}\right|^{q}$ is prequasiinvex on $K$ for $q>1$ we have

$$
\begin{aligned}
& \left|\frac{b-a}{2}\left(\frac{2 a+b}{3}\right) f^{\prime \prime}(b)-\frac{a+b}{2} f^{\prime}(b)+\frac{f(b) b-f(a) a}{b-a}-\frac{1}{b-a} \int_{a}^{b} f(x) d x\right| \\
\leq & \frac{3^{\frac{1}{q}}}{2}(b-a)^{1+\frac{1}{p}}\left(\frac{1}{2 p+1}\right)^{\frac{1}{p}}\left(\max \left\{\left|f^{\prime \prime \prime}(a)\right|^{q},\left|f^{\prime \prime \prime}(b)\right|^{q}\right\}\right)^{\frac{1}{q}} C^{\frac{1}{q}}(q, a, b)
\end{aligned}
$$

where

$$
C(q, a, b)= \begin{cases}\frac{b-a}{3} L_{q}^{q}\left(\frac{b+2 a}{3}, a\right), & a>0, \frac{b+2 a}{3}>0 \\ \frac{2}{q+1} A\left[\left(\frac{b+2 a}{3}\right)^{q+1},(-a)^{q+1}\right], & a<0, \frac{b+2 a}{3}>0, \\ \frac{b-a}{3} L_{q}^{q}\left(-a,-\frac{b+2 a}{3}\right), & a<0, \frac{b+2 a}{3}<0 .\end{cases}
$$

Remark 2.14. If the mapping $\eta$ satisfies condition $\mathrm{C}$ then by use of the prequasiinvexity of $\left|f^{\prime \prime \prime}\right|^{q}$ we get

$$
\begin{aligned}
\left|f^{\prime \prime \prime}(a+t \eta(b, a))\right|^{q} & =\left|f^{\prime \prime \prime}(a+\eta(b, a)+(1-t) \eta(a, a+\eta(b, a)))\right|^{q} \\
& \leq \max \left\{\left|f^{\prime \prime \prime}(a)\right|^{q},\left|f^{\prime \prime \prime}(a+\eta(b, a))\right|^{q}\right\}
\end{aligned}
$$

for every $t \in[0,1]$. If we use the inequality $(12)$ in the proof of Theorem 2.12 , then the inequality (11) becomes the following inequality:

$$
\left|I_{f}(a, b, \eta)\right|
$$




$$
\leq \frac{3^{\frac{1}{q}}}{2} \eta^{2+\frac{1}{p}}(b, a)\left(\frac{1}{2 p+1}\right)^{\frac{1}{p}}\left(\max \left\{\left|f^{\prime \prime \prime}(a)\right|^{q},\left|f^{\prime \prime \prime}(a+\eta(b, a))\right|^{q}\right\}\right)^{\frac{1}{q}} C_{\eta}^{\frac{1}{q}}(q, a, b)
$$

We note that by use of the prequasiinvexity of $\left|f^{\prime \prime \prime}\right|^{q}$ we have $\left|f^{\prime \prime \prime}(a+\eta(b, a))\right|^{q} \leq$ $\max \left\{\left|f^{\prime \prime \prime}(a)\right|^{q},\left|f^{\prime \prime \prime}(a+\eta(b, a))\right|^{q}\right\}$. Therefore, the inequality 14 is better than the inequality (11).

Theorem 2.15. Let $K \subseteq \mathbb{R}$ be an open invex subset with respect to mapping $\eta(\cdot, \cdot): K \times K \rightarrow \mathbb{R}^{n}$ and $a, b \in K$ with $\eta(b, a)>0$. Suppose that the function $f: K \rightarrow \mathbb{R}$ is a three times differentiable function on $K$ such that $f^{\prime \prime \prime} \in L[a, a+\eta(b, a)]$. If $\left|f^{\prime \prime \prime}\right|^{q}$ is prequasiinvex on $K$ for $q \geq 1$, then the following inequality holds:

$$
\left|I_{f}(a, b, \eta)\right| \leq \frac{27}{2}\left(\max \left\{\left|f^{\prime \prime \prime}(a)\right|^{q},\left|f^{\prime \prime \prime}(b)\right|^{q}\right\}\right)^{\frac{1}{q}} D_{1, \eta}(a, b)
$$

where

$$
D_{1, \eta}(a, b):=\left\{\begin{array}{lr}
\frac{\eta^{3}(b, a)}{27} \frac{\eta(b, a)+4 a}{12}, & a>0, \beta>0 \\
\frac{\eta^{3}(b, a)}{27} \frac{\eta(b, a)+4 a}{12}+\frac{a^{4}}{6}, & a<0, \beta>0 \\
-\frac{\eta^{3}(b, a)}{27} \frac{\eta(b, a)+4 a}{12}, & a<0, \beta<0
\end{array},\right.
$$

Proof. From Lemma 2.1 and Power-mean integral inequality, we obtain

$$
\begin{aligned}
& \left|I_{f}(a, b, \eta)\right| \\
\leq & \frac{1}{2} \eta^{3}(b, a) \int_{0}^{1} t^{2}\left|\beta_{t}\right|\left|f^{\prime \prime \prime}(a+t \eta(b, a))\right| d t \\
\leq & \frac{1}{2} \eta^{3}(b, a)\left(\int_{0}^{1} t^{2}\left|\beta_{t}\right| d t\right)^{1-\frac{1}{q}}\left(\int_{0}^{1} t^{2}\left|\beta_{t}\right|\left|f^{\prime \prime \prime}(a+t \eta(b, a))\right|^{q} d t\right)^{\frac{1}{q}} \\
\leq & \frac{1}{2} \eta^{3}(b, a)\left(\int_{0}^{1} t^{2}\left|\beta_{t}\right| d t\right)^{1-\frac{1}{q}}\left(\int_{0}^{1} t^{2}\left|\beta_{t}\right| \max \left\{\left|f^{\prime \prime \prime}(a)\right|^{q},\left|f^{\prime \prime \prime}(b)\right|^{q}\right\} d t\right)^{\frac{1}{q}} \\
= & \frac{1}{2} \eta^{3}(b, a)\left(\max \left\{\left|f^{\prime \prime \prime}(a)\right|^{q},\left|f^{\prime \prime \prime}(b)\right|^{q}\right\}\right)^{\frac{1}{q}} \int_{0}^{1} t^{2}\left|\beta_{t}\right| d t \\
= & \frac{27}{2}\left(\max \left\{\left|f^{\prime \prime \prime}(a)\right|^{q},\left|f^{\prime \prime \prime}(b)\right|^{q}\right\}\right)^{\frac{1}{q}} \int_{a}^{\beta}(x-a)^{2}|x| d x \\
= & \frac{27}{2}\left(\max \left\{\left|f^{\prime \prime \prime}(a)\right|^{q},\left|f^{\prime \prime \prime}(b)\right|^{q}\right\}\right)^{\frac{1}{q}} D_{1, \eta}(a, b) .
\end{aligned}
$$

This completes the proof of theorem.

Corollary 2.16. Suppose that all the assumptions of Theorem 2.15 are satisfied. If we choose $\eta(b, a)=b-a$ then when $\left|f^{\prime \prime \prime}\right|^{q}$ is prequasiinvex on $K$ for $q \geq 1$ we have

$$
\left|\frac{b-a}{2}\left(\frac{2 a+b}{3}\right) f^{\prime \prime}(b)-\frac{a+b}{2} f^{\prime}(b)+\frac{f(b) b-f(a) a}{b-a}-\frac{1}{b-a} \int_{a}^{b} f(x) d x\right|
$$




$$
\leq \frac{27}{2} \frac{D_{1}(a, b)}{b-a}\left[\max \left\{\left|f^{\prime \prime \prime}(a)\right|^{q},\left|f^{\prime \prime \prime}(b)\right|^{q}\right\}\right]^{\frac{1}{q}}
$$

where

$$
D_{1}(a, b)=\left\{\begin{array}{lr}
\frac{(b-a)^{3}}{27} \frac{b+3 a}{12}, & a>0, \frac{b+2 a}{3}>0 \\
\frac{(b-a)^{3}}{27} \frac{b+3 a}{12}+\frac{a^{4}}{6}, & a<0, \frac{b+2 a}{3}>0 \\
-\frac{(b-a)^{3}}{27} \frac{b+3 a}{12}, & a<0, \frac{b+2 a}{3}<0
\end{array},\right.
$$

Remark 2.17. If we use the inequality 12 in the proof of Theorem 2.15, then the inequality (14) becomes the following inequality:

$$
\left|I_{f}(a, b, \eta)\right| \leq \frac{27}{2}\left(\max \left\{\left|f^{\prime \prime \prime}(a)\right|^{q},\left|f^{\prime \prime \prime}(a+\eta(b, a))\right|^{q}\right\}\right)^{\frac{1}{q}} D_{1, \eta}(a, b)
$$

This inequality is better than the inequality (14).

Corollary 2.18. If we take $q=1$ in Theorem 2.15, then we have the following inequality:

$$
\left|I_{f}(a, b, \eta)\right| \leq \frac{27}{2} \max \left\{\left|f^{\prime \prime \prime}(a)\right|^{q},\left|f^{\prime \prime \prime}(b)\right|^{q}\right\} D_{1, \eta}(a, b)
$$

\section{Competing Interests}

The author(s) do not have any competing interests in the manuscript.

\section{REFERENCES}

1. Pečarić JE, \& Tong, Y. L. (1992). Convex functions, partial orderings, and statistical applications. Academic Press.

2. Dragomir, S. S., \& Pearce, C. E. M. (2000). Selected Topics on Hermite-Hadamard Inequalities and Applications, RGMIA Monographs, Victoria University, 2000. ONLINE: http://rgmia. vu. edu. au/monographs.

3. İşcan İ., Set, E., \& zdemir, M. E. (2014). On new general integral inequalities for s-convex functions. Applied Mathematics and Computation, 246, 306-315.

4. İşcan İ, Kadakal, H., \& Kadakal, M. (2017)., Some New Integral Inequalities for $n$ - Times Differentiable Quasi-Convex Functions. Sigma Journal of Engineering and Natural Sciences, 35 (3), 363-368.

5. Maden, S., Kadakal, H., Kadakal, M., \& İşcan İ. (2017) Some new integral inequalities for $n$-times differentiable convex functions. J. Nonlinear Sci. Appl , 10 (12), 6141-6148.

6. Hadamard, J. (1893). tude sur les proprits des fonctions entires et en particulier d'une fonction considre par Riemann. Journal de mathmatiques pures et appliques, 171-216.

7. Ion, D. A. (2007). Some estimates on the Hermite-Hadamard inequality through quasiconvex functions. Annals of the University of Craiova-Mathematics and Computer Science Series,34, 82-87.

8. Weir, T., \& Mond, B. (1988). Pre-invex functions in multiple objective optimization. Journal of Mathematical Analysis and Applications, 136(1), 29-38.

9. Mohan, S. R., \& Neogy, S. K. (1995). On invex sets and preinvex functions. Journal of Mathematical Analysis and Applications, 189(3), 901-908.

10. Yang, X. M., Yang, X. Q., \& Teo, K. L. (2003). Generalized invexity and generalized invariant monotonicity. Journal of Optimization Theory and Applications, 117(3), 607625 .

11. Antczak, T. (2005). Mean value in invexity analysis. Nonlinear Analysis: Theory, Methods \& Applications, 60(8), 1473-1484. 
12. Barani, A., Ghazanfari, A. G., \& Dragomir, S. S. (2011). Hermite-Hadamard inequality through prequsiinvex functions. RGMIA Res. Rep. Collect, 14.

13. Ben-Israel, A., \& Mond, B. (1986). What is invexity?. The ANZIAM Journal, 28(1), 1-9.

14. Noor, M. A. (2005). Invex equilibrium problems. Journal of Mathematical Analysis and Applications, 302(2), 463-475.

15. Aslam Noor, M. (1994). Variational-like inequalities. Optimization, 30(4), 323-330.

16. Pini, R. (1991). Invexity and generalized convexity. Optimization, 22(4), 513-525.

17. Yang, X. M., \& Li, D. (2001). On properties of preinvex functions. Journal of Mathematical Analysis and Applications, 256(1), 229-241.

18. Noor, M. A. (2007). Hermite-Hadamard integral inequalities for log-preinvex functions. $J$. Math. Anal. Approx. Theory, 2(2), 126-131.

19. Barani, A., Ghazanfari, A. G., \& Dragomir, S. S. (2012). Hermite-Hadamard inequality for functions whose derivatives absolute values are preinvex. Journal of Inequalities and Applications, 2012(1), 247.

20. İşcan İ., (2015). Ostrowski type inequalities for functions whose derivatives are preinvex. Bulletin of the Iranian Mathematical Society, 40(2), 373-386.

21. Latif, M. A., \& Dragomir, S. S. (2013). Some Hermite-Hadamard type inequalities for functions whose partial derivatives in absloute value are preinvex on the co-oordinates. Facta Universitatis (NIS) Ser. Math. Inform, 28(3), 257-270.

22. Matloka M., (2014) On some new inequalities for differentiable $\left(h_{1} ; h_{2}\right)$-preinvex functions on the co-ordinates. Mathematics and Statistics, 2(1), 6-14.

Huriye Kadakal

Institute of Science, Ordu University-Ordu-Türkiye.

e-mail: huriyekadakal@hotmail.com

Mahir Kadakal

Department of Mathematics, Faculty of Sciences and Arts, Giresun University-GiresunTürkiye.

e-mail: mahirkadakal@gmail.com

İmdat İşcan

Department of Mathematics, Faculty of Sciences and Arts, Giresun University-GiresunTürkiye.

e-mail: imdat.iscan@giresun.edu.tr 